\title{
تقدير الخصائص الفيزوكيميائية والحسية لصمغ بذور الريحان Ocimum basilicum العراقي والكشف عن بعض المركبات الفعالة فيه
}

\section{Determination of the Physicochemical and Organoleptic Properties of Iraqi Basil Seeds Gum (Ocimum basilicum) and Detection of Phytochemicals}

\author{
ميسون ظافر هادي العاني

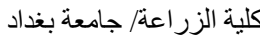 \\ كلية الزر اعة/ جامعة جامعة بغداد \\ Inas Mudhafar Khalil Al-Aubadi Mayson Thaafir Hadi Al-Ani \\ إيناس مظفر خليل العبادي \\ College of Agriculture/ Baghdad University \\ College of Agriculture/ Baghdad University
}

E-mail: inas_alaubadi@yahoo.com

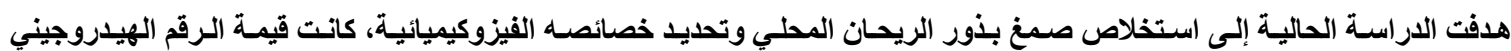

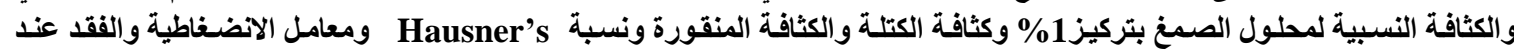

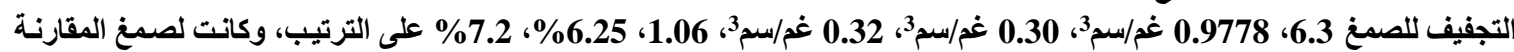

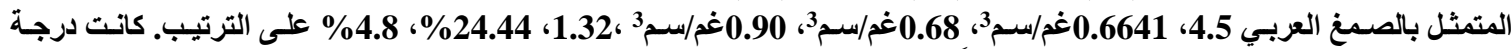

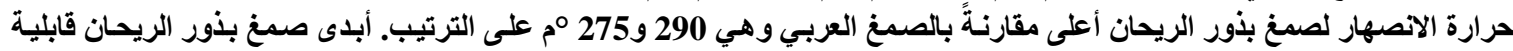

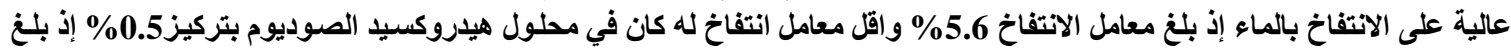

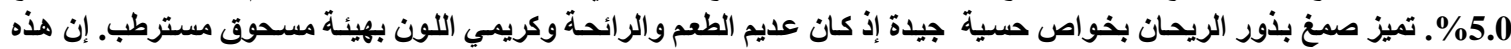

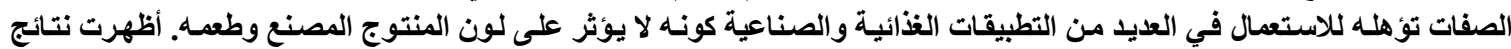

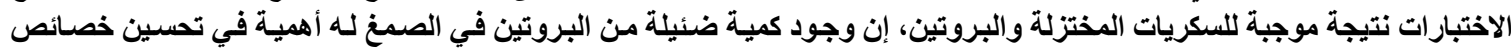
الاستحلاب وربط الاهن وبعض الصفات الوظيفية الأخرى.

الكلمات الدالة: بذور الريحان، صمغ، الخصائص الفيزوكيميائية، الخصائص الحسية، المركبات الفعالة

Abstract

The aim of this study was to extract basil seed gum and to examine the physicochemical properties. The $\mathrm{pH}$ value, relative density of $1 \%$ gum solution, bulk density, tap density, Hausner's ratio, compressibility index and loss on drying of the gum are: $6.3,0.977 \mathrm{~g} / \mathrm{cm}^{3}, 0.30 \mathrm{~g} / \mathrm{cm}^{3}, 0.32 \mathrm{~g} / \mathrm{cm}^{3}, 1.06$, $6.25 \%$ and $7.2 \%$ respectively, and for the Arabic gum as a comparison gum were: $4.5,0.6641 \mathrm{~g} / \mathrm{cm}^{3}$, $0.68 \mathrm{~g} / \mathrm{cm}^{3}, 0.90 \mathrm{~g} / \mathrm{cm}^{3}, 1.32,24.44 \%, 4.8 \%$ respectively. The melting point of the basil seed gum was high compared to the Arabic gum which was 290 and $275{ }^{\circ} \mathrm{C}$ respectively. The gum shows high swelling ability with water where the swelling index reached $5.6 \%$ and it was less than the swelling index in $0.5 \%$ concentration of sodium hydroxide solution reaching $5.0 \%$. Basil seed gum characterized as a good organoleptic properties as it was tasteless and odorless, creamy color shaped like hygroscopic powder. It also shows positive results for reducing sugar and protein. The presence of small amount of protein in gum was very important for enhancing emulsification properties, fat holding capacity and other functional properties.

Key wards: Basil seed, gum, physiochemical properties, organoleptic properties, Phytochemicals

\footnotetext{
تمتلك الاصماغ و الهلامات العديد من النطبيقات في المجالات الذذائية والطبية والصيدلانية إذ تعد من المو اد الآمنة صحيا كونها غير السامة

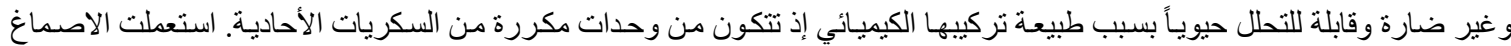

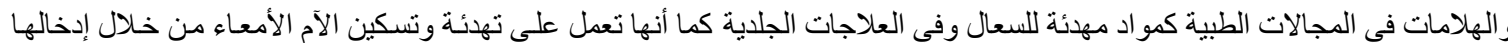

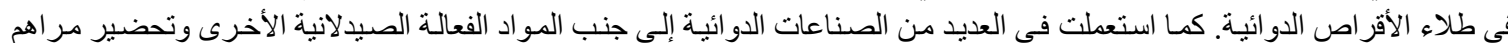

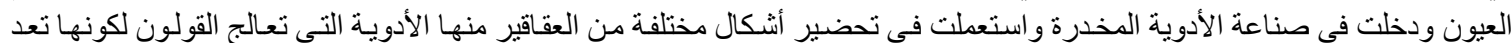

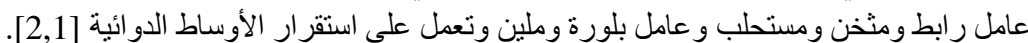

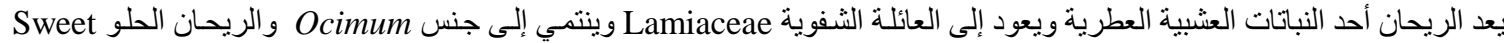
من basil
} 
معظم أنواع الريحان نباتات عشبية حولية وبعضها متحملة لظروف المناخ الحار في المناطق الاستو ائية مثل الريحان المقدس و الريحان

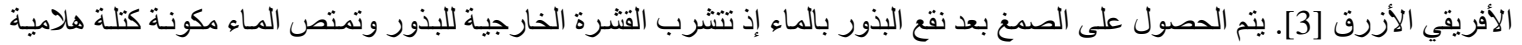

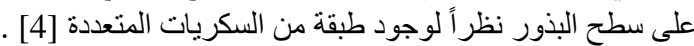

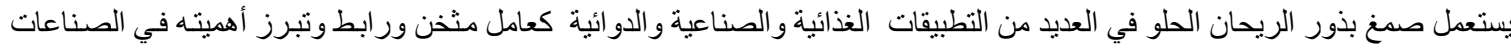

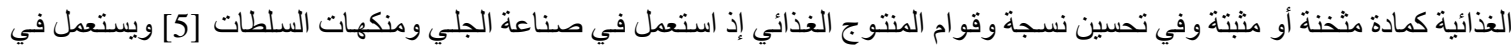

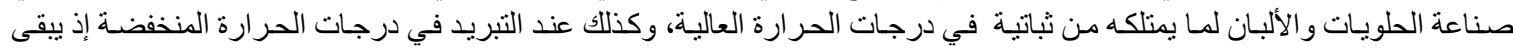

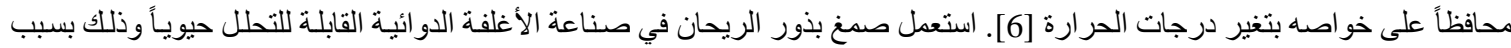

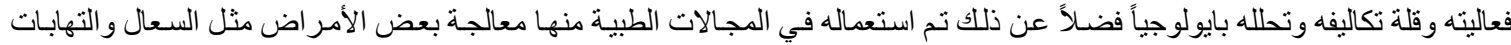

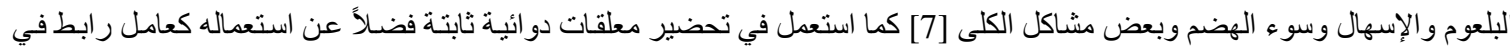

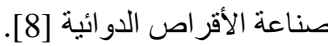

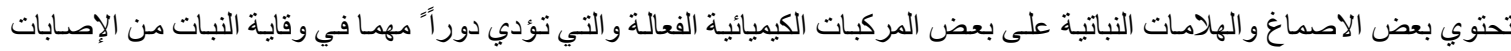

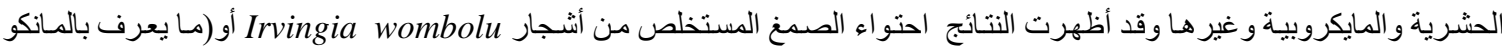

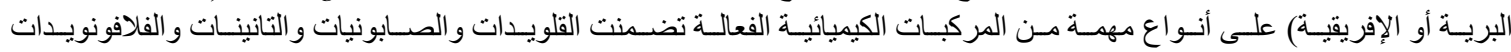

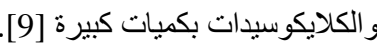

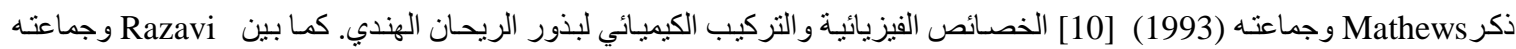

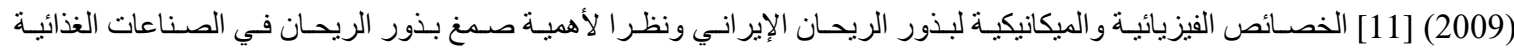

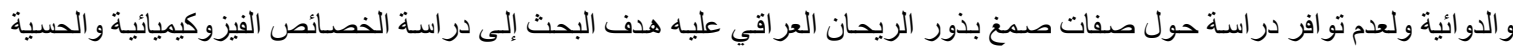

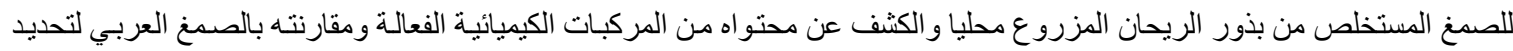

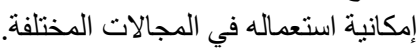
المواد وطرائق العمل

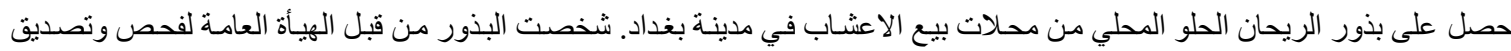

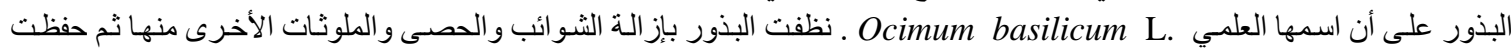
بظروف باردة وجافة في قناني زجاجية نظيفة محكمة الغلق لحين استعمالها في البحث.

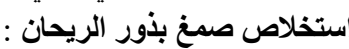

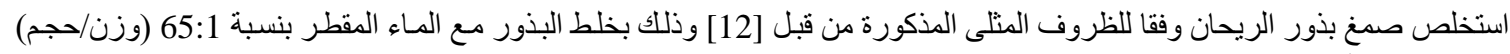

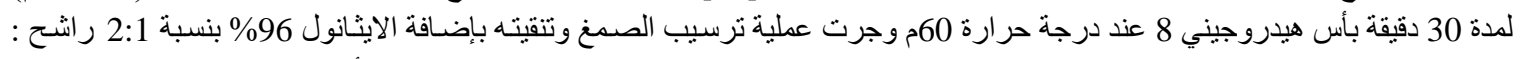

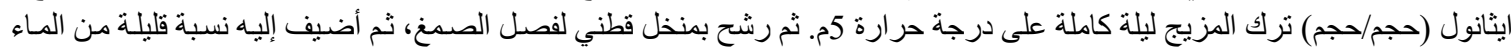

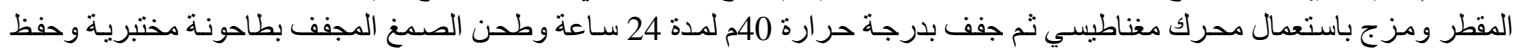
المسحوق في أو عية محكمة الغلق. تقدير الخصائص الفيزوكيميائية لصمغ بذونة الصور الريحان والصمغ العربي

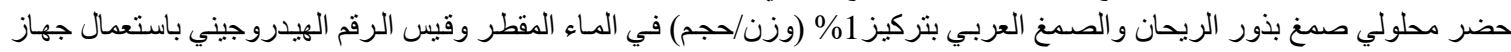

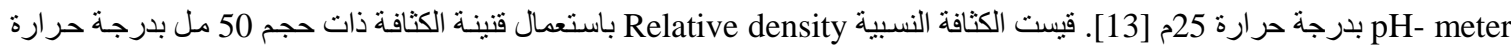

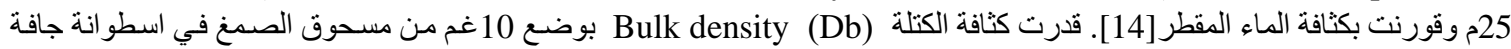

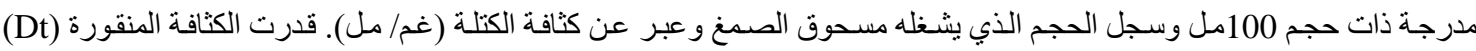
Tapped density للطريقة التي ذكر ها Muazu وجماعته (2014) [15] وحسب المعادلات الآتية:

\section{كثافة الكتلة = وزن مسحوق الصمغ/ الحجم الأي يشغله مسحوق الصمغ}

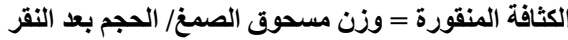

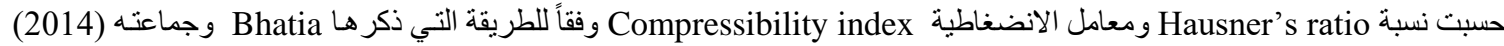

\section{نسبة Hausner's= الكثافة المنقورة/كثافة الكتلة}

[16] وفقاً للمعادلات الآتية:

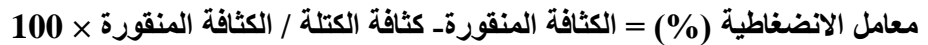

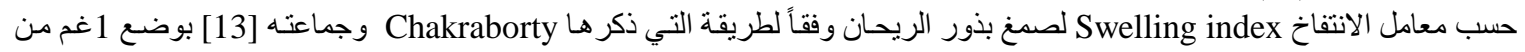

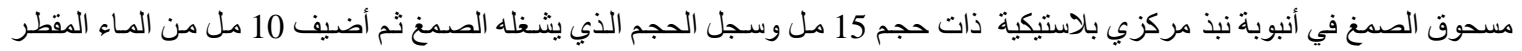

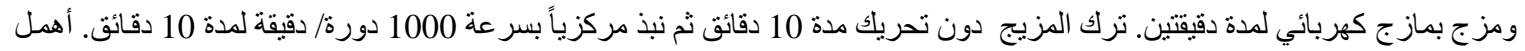

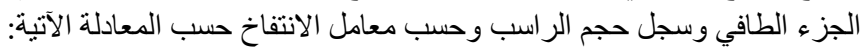

قابلية الانتفاخ (\%) = حجم المادة بعد الانتفاخ - حجم المادة قبل الانتفاخ/ حجم المادة قبل الانتفاخ × 100 
كررت التجربة باستعمال محلول حامض الهيدروكلوريك بتركيز 0.1 عياري ومحلول كلوريد الصوديوم بتركيز 0.9 \% ومحلول هيدروكسيد الصوديوم بتركيز 0.5 عياري.

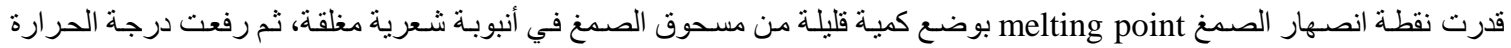

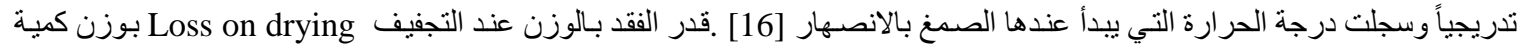

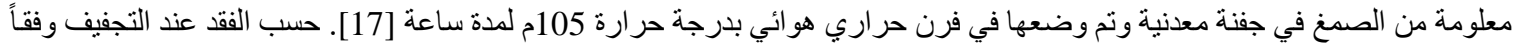
للمعادلة الآتية:

$$
\text { الفقد عند التجفيف (\%) = الوزن الأولي - الوزن النهائي/ الوزن الأولي ×100 }
$$

تعيين الخصائص الحسية للصمغ

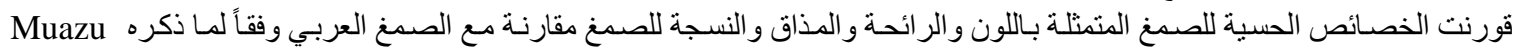
وجماعته [15].

الكثف عن المركبات الكيميائية الفعالة في صمغ بذور الريحان

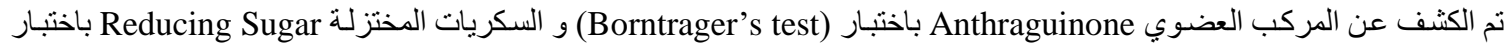

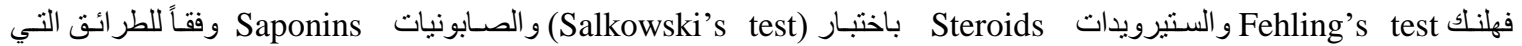

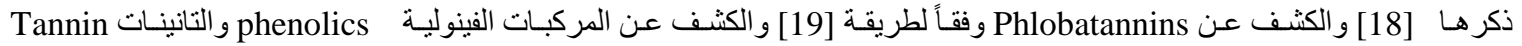

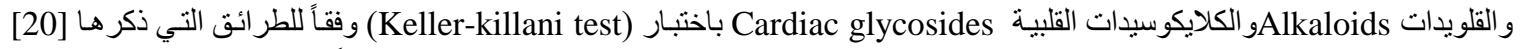
و الكثف عن الفلافونويدات Flavonoids وفقاً للطريقة التي ذكر ها [21] و الكثف عن البروتين وفقا لطريقةً [22] . النتائج والمناقشة الخواص الفيزوكيميائية لصمغ بذور الريحان والصمغ العربي

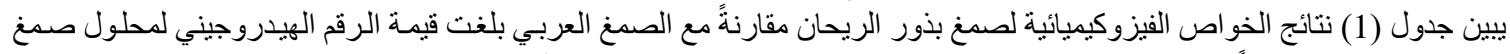

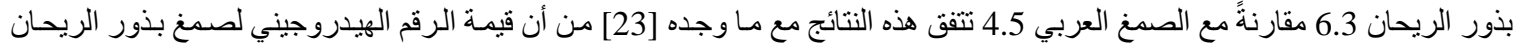

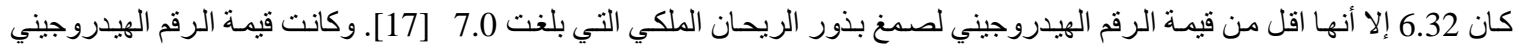

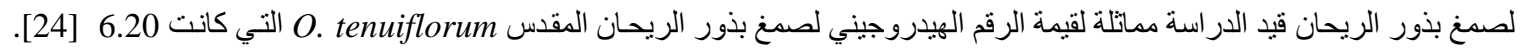

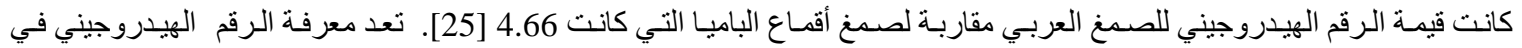
التركيبات المعدة للتطبيقات الصيدلانية معيار أ مهماً وضرورياً جداً في تحديد مدى ملائمته للتطبيقات المختلفة وذللك لان الثباتية و الفعالية الفسيولوجية تعتمد على الرقم الهيدروجيني [26]. جدول (1): الخصائص الفيزوكيمائية لصمغ بذور الريحان والصمغ العربي

\begin{tabular}{|c|c|c|}
\hline الصمغ العربي & صمغ بذور الريحان & الخصائص \\
\hline 4.5 & 6.3 & الرقم الهياروجيني لمحلول الصمغ 1\% \\
\hline 0.6441 & 0.9778 & الكثافة النسبية (غم/سم33) لمحلول الصمغ 1\% \\
\hline 0.68 & $\mathbf{0 . 3 0}$ & كثافة الكتلة (غم/سم3) \\
\hline 0.90 & 0.32 & الكثافة المنقورةٌ (غم/سم3) \\
\hline 1.32 & 1.06 & نسبة Hausner's \\
\hline 24.44 & 6.25 & معامل الانضغاطية (\%) \\
\hline 4.8 & 7.2 & الفقا عند التجفيف (و/ و) (\%) \\
\hline 275 & 290 & درجة الانصهار (ْم) \\
\hline
\end{tabular}

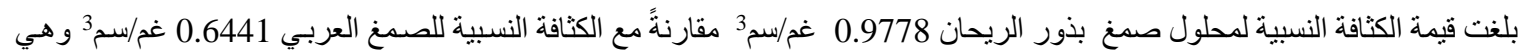

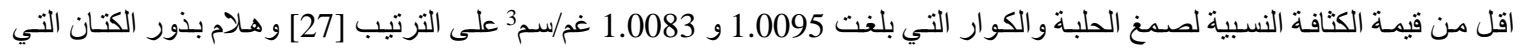

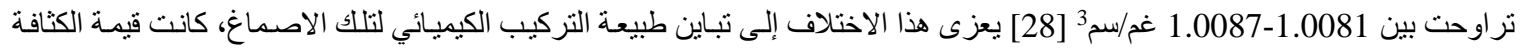

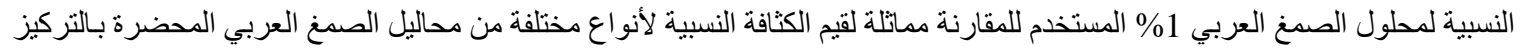
نفسه من اشجار

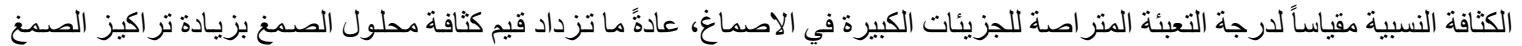

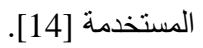

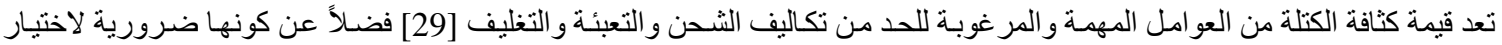

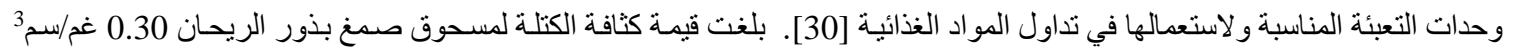

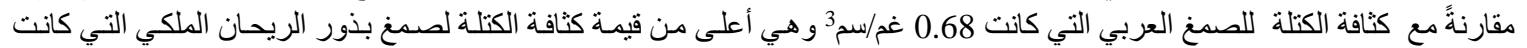

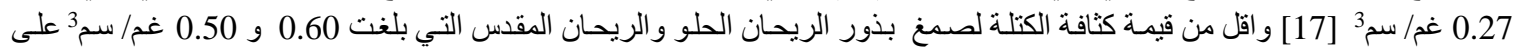

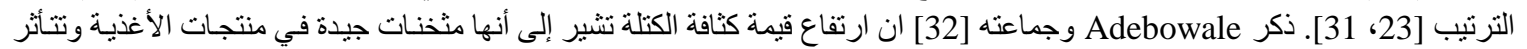

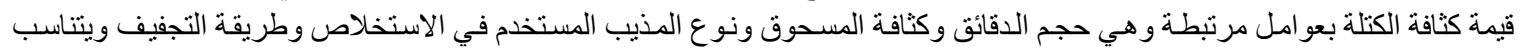

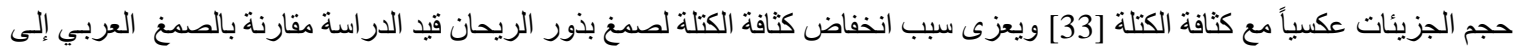

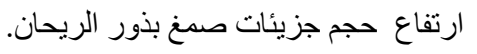




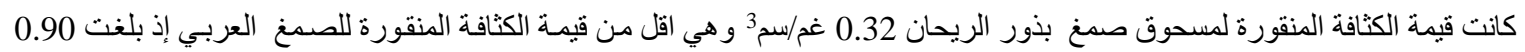

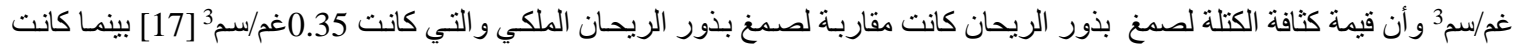

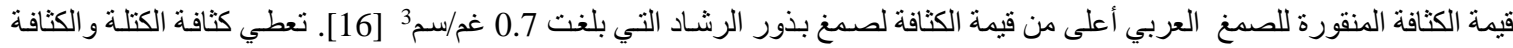

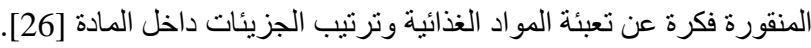

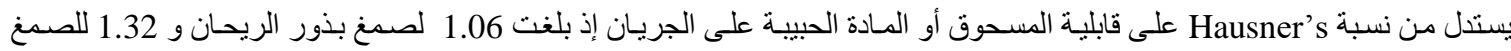

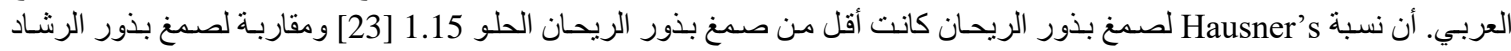

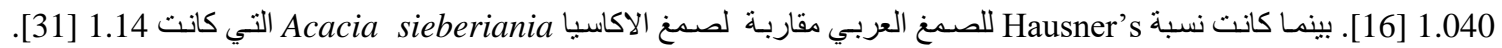

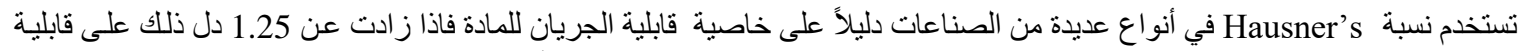

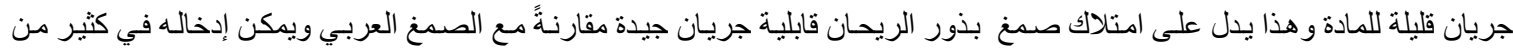

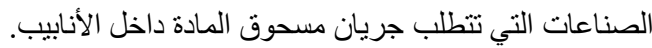

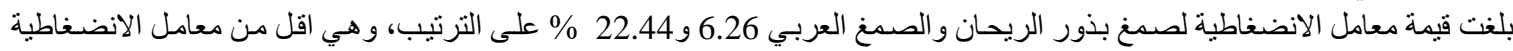

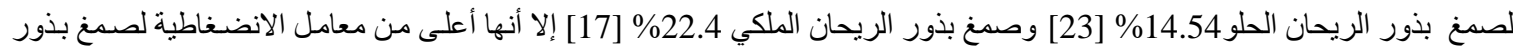

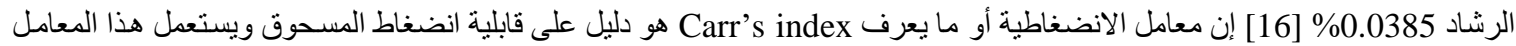

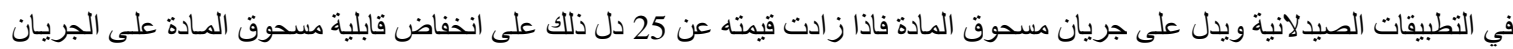

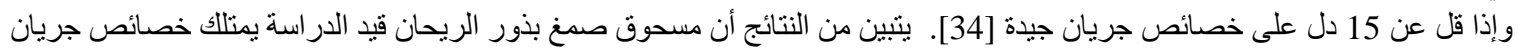

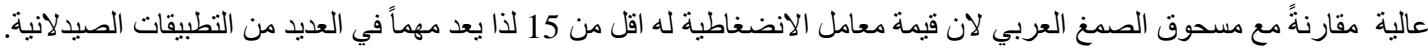

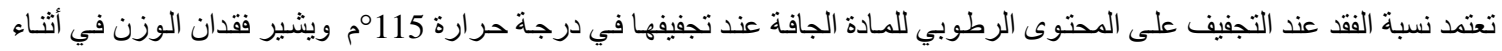

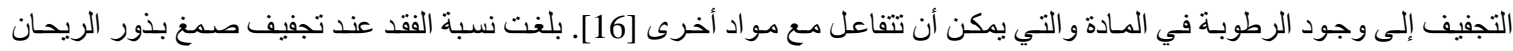

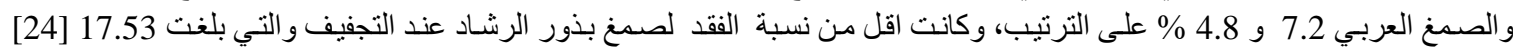

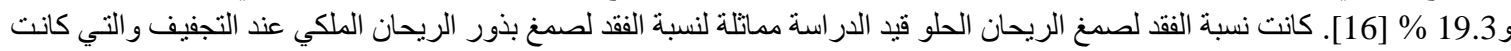

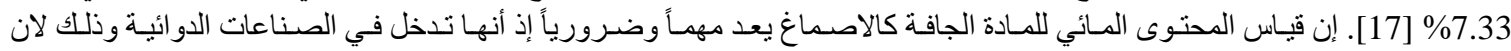

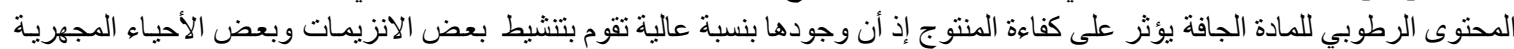

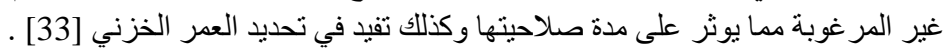

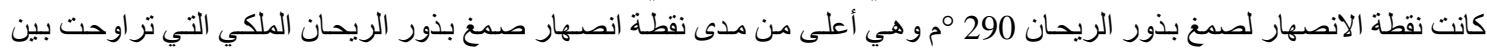

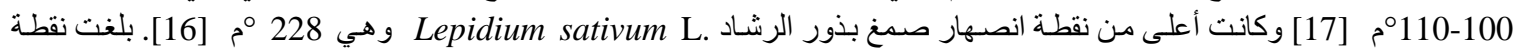

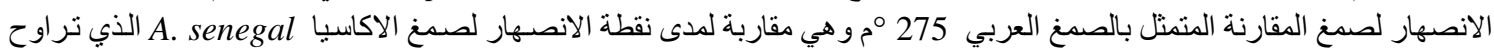

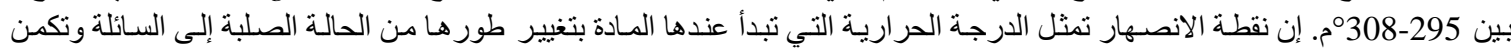

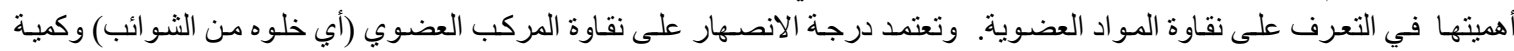

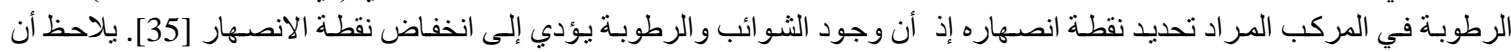

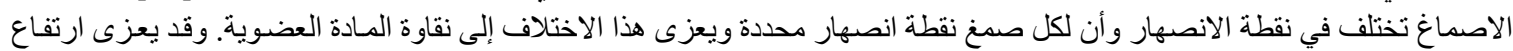

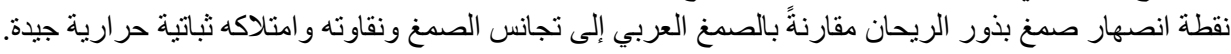
تقدير معامل الانتفاخ لصمغ بذور بذور الريحان

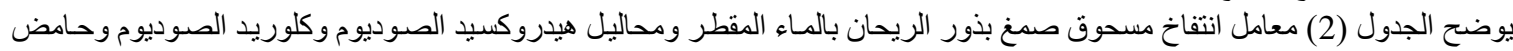

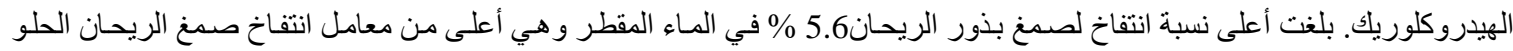

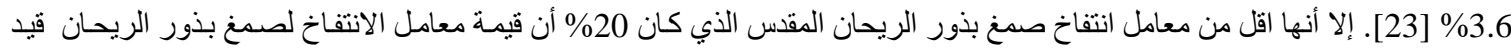

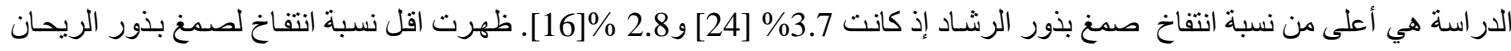

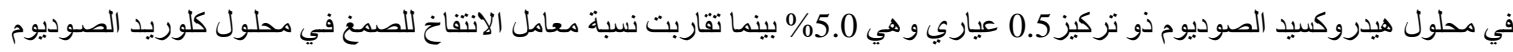

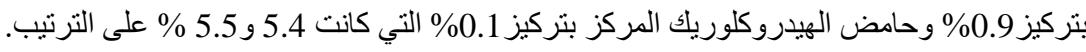

\begin{tabular}{|c|c|}
\hline معامل الانتفاخ \% & المحاليل \\
\hline 5.6 & الماء المقطر \\
\hline 5.0 & هيدروكسيد الصوديوم (NaOH) بتركيز 0.5 عياري \\
\hline 5.4 & كلوريد الصوديوم (NaCl) بتركيز 0.9 \% \\
\hline 5.5 & حامض الهيلروكلوريك (HCl) بتركيز 0.1 عياري \\
\hline
\end{tabular}

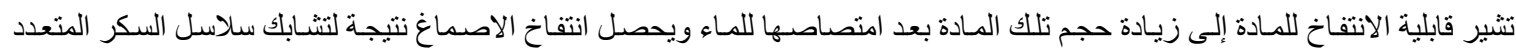

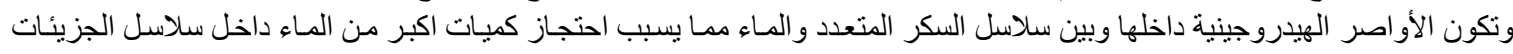

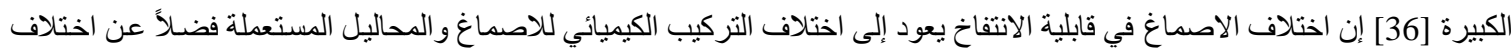

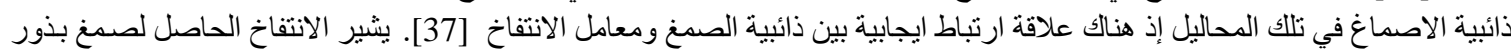

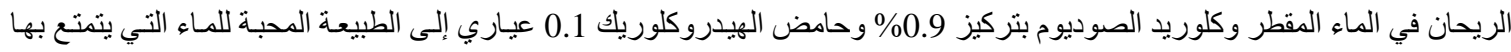


الصمغ و التي يمكن استغلالها لتصميم أثنكال الدواء المختلفة. وتبرز أهمية ارتفاع قيمة معامل الانتفاخ للصمغ إلى إمكانيـة استعماله بوصـفـه

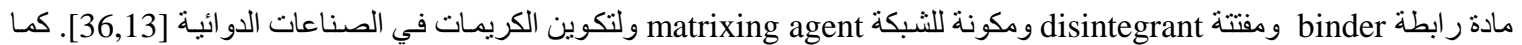

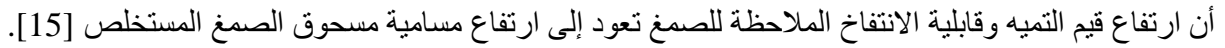

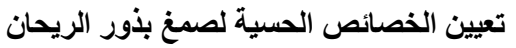

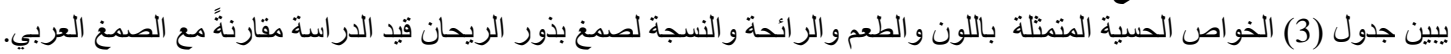
جدول (3): الصفات الحسية لأنموذجي صمغ بذور الريحان والصمغ العربي

\begin{tabular}{|c|c|c|c|c|}
\hline النسجة & الرائحة & الطعم & اللون & النموذج \\
\hline مسحوق مسترطب & عديم الرائحة & عديم الطعم & كريمي فاتح & صمغ بذور الريحان \\
\hline مسحوق ناعم & عديم الرائحة & عديم الطعم & كريمي & الصمغ العربي \\
\hline
\end{tabular}

تميز صمغ بذور الريحان بلون كريمي فاتح مقارنةً مع الصمغ العربي الذي كان كريمي اللون وقد أمتاز صمغي بذور الريحان و الصمغ الريخ

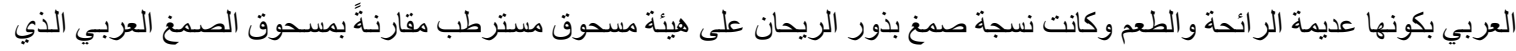

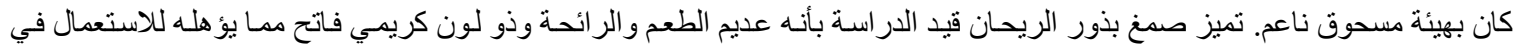

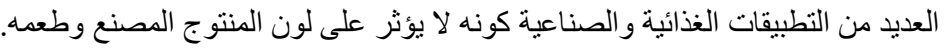

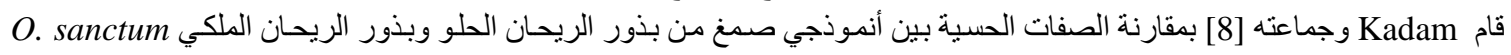

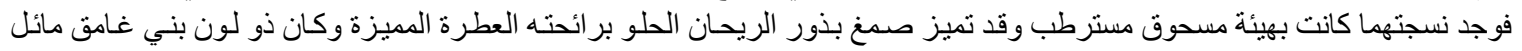

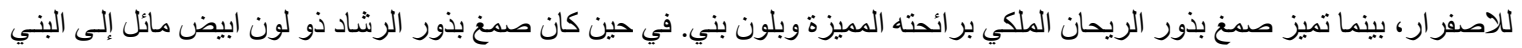

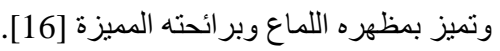

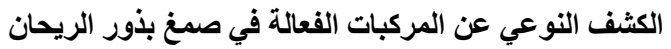

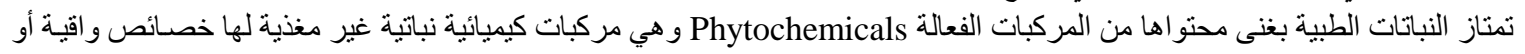

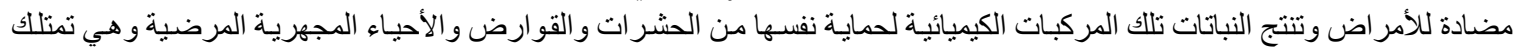

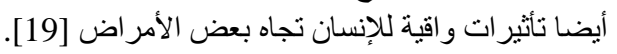

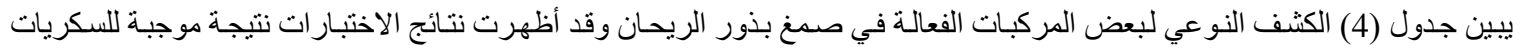

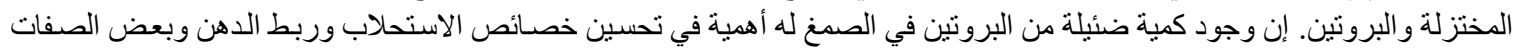

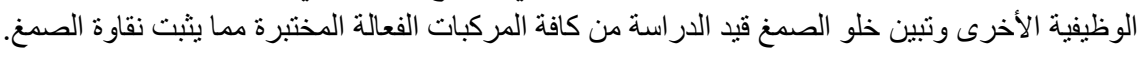

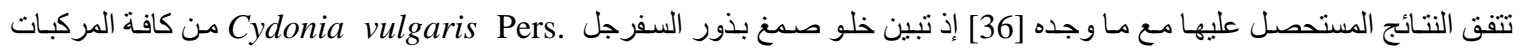

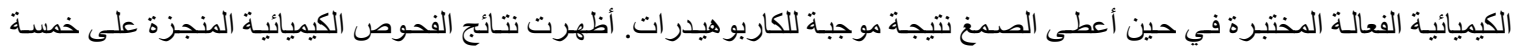

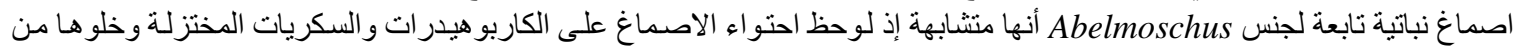
البرونين، ومن بين النو اتج الايضية الثانوية المختبرة تبين وجود الفلافونويدات والكومارين و الصابونيات و غياب التانينات [25]. جدول (4): الكثف النوعي عن المركبات الفعالة في صمغ بذور الريحان

\begin{tabular}{|c|c|}
\hline نتيجة الاختبار & نوع الاختبار \\
\hline- & اختبار الأنثراكينون (Borntrager's test) \\
\hline - & Phlobatannins اختبار \\
\hline+ & اختبار السكريات المختزلة Reducing Sugar (Fehling’s test) \\
\hline - & Steroids (Salkowski’s test) اختبار الستيرويدات \\
\hline- & Saponins test اختبار الصابونيات \\
\hline - & Phenolics test اختبار المركبات الفينولية \\
\hline - & Tannins test اختبار التانينات \\
\hline - & Cardiac glycosides (Keller-Killani test) اختبار الكلايكوسيدات القلبية \\
\hline- & Flavonoids test الكشف عن الفلافونويدات \\
\hline - & Alkaloids test اختبار القلويدات \\
\hline+ & protein test اختبار البروتين \\
\hline
\end{tabular}

نستنتج من البحث تجانس صمغ بذور الريحان ونقاوته وامتلاكه ثباتية حر ارية جيدة وخصائص جريان عالية مما يشير إلى إمكانية استعماله

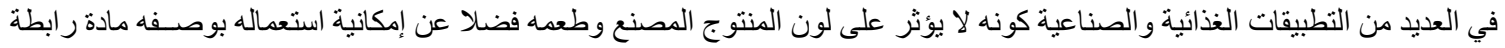

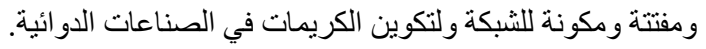


1. Bhosale, R. R., Osmani, R. A. M. and Moin, A. (2014). Natural gums and mucilages: a review on multifaceted excipients in pharmaceutical science and research. International Journal of Pharmacognosy and Phytochemical Research. 6(4): 901-912.

2. Jani, G. K., Shah, D. P., Prajapati, V. D. and Jain, V. C. (2009). Gums and mucilages: versatile excipients for pharmaceutical formulations. Asian Journal of Pharmaceutical Sciences. 4 (5): 308-322.

3. Jacqueline, A. S. (2001). Father kino's herbs: growing \& using them today. Tierra Del. Sol. Institute Press, Tucson,USA.

4. Azoma, J. and Sakamoto, M. (2003). Cellulosic hydrocolloid system present in seed of plants. Trends in Glycoscience and Glycotechnology. 15: 1-14.

5. Hosseini-Parvar, S. H., Matia- merino, L., Goh, K. K. T. and Mortazavi, S. A. (2010). Steady shear flow behavior of gum extracted from Ocimum basilicum L. seed: effect of concentration and temperature. Journal of Food Engineering. 101: 236-243.

6. Rafe, A. and Razavi, S. M. A. (2013). Dynamic viscoelastic study on the gelation of basil seed gum. International Journal of Food Science and Technology. 48: 556-563.

7. Vieira, R. F. and Simon, J. E. (2000). Chemical characterization of basil (Ocimum spp.) found in the market and used in traditional medicine in Brazil. Economic Botany. 54(2): 207-216.

8. Kadam, P. V., Yadav, K. V., Jagdale, S. K., Shivatare, R. S., Bhilwade, S. and Manohar, J. (2012). Evaluation of Ocimum sanctum and Ocimum basillicum mucilage- as a pharmaceutical excipient. Journal of Chemical and Pharmaceutical Research. 4(4):1950-1955.

9. Ikechukwu, O. V. and Salome, C. A. (2013). Physicochemical characterisation of Irvingia wombolu gum in tramadol encapsulated granules onyishi. African Journal of Pharmacy and Pharmacology. 7(42): 27882793.

10. Mathews, S., Singhal, R. S. and Kulkarni, P. R. (1993). Ocimum basilicum: a new non-conventional source of fiber. Food Chemistry. 47: 399-401.

11. Razavi, S. M. A., Mortazavi, S. A., Matia-Merino, L., Hosseini-Parvar, S. H., Motamed zadegan, A. and Khanipour, E. (2009). Optimization study of gum extraction from basil seeds (Ocimum basilicum L.). International Journal of Food Science and Technology. 44: 1755-1762. 12. العبـادي، إيناس مظفر خليل والعاني، ميسون ظـافر هـادي. (2015). دراسـة الظروف المثلى لاستخلاص صمخ بذور الريحسان المحلي.

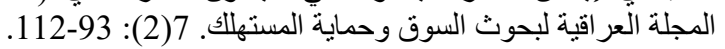

13. Chakraborty, J. and Dash, S. (2014). Optimization and characterization of purified gummy polysaccharide isolated from Aegle marmelos fruit pulp as a novel pharmaceutical excipient. International Journal of Pharmacy and Pharmaceutical Sciences. 6(1): 0975-1491.

14. Yusuf, A. K. (2011). Studies on some physicochemical properties of the plant gum exudates of Acacia senegal (Dakwara), Acacia sieberiana (Farar kaya) and Acacia nilotica (Bagaruwa). Journal of Research in National Development. 9, 1596 - 8308.

15. Muazu, J., Alpha, A. and Mohammed, G.T.(2014). Isolation and release retardant properties of a plant gum obtained from ayoyo. Caribbean Journal of Science and Technology. 2: 301-313.

16. Bhatia, N. M., Salunkhe, S. S., Mali, S. S., Gadkari, S. S., Hajare, A. A., Gaikwad, S. V. and Karade, R. S. (2014). Extraction and characterization of mucilage from Lepidium sativum Linn. seeds. Scholars Research Libraryder Pharmacia Lettre. 6(1):65-70.

17. Kumar, M. B., Bharath, S., Deveswaran, R., Basavaraj, B. V. and Madhavan, V. (2013). Isolation and evaluation of suspending property of Holy basil seed mucilage. Journal of Advanced Pharmaceutical Research. 4(3): 64- 68.

18. Aiyelaagbe, O. O. and Osamudiamen, P. M. (2009). Phytochemical screening for active compounds in Mangifera indica leaves from Ibadan, Oyo State. Plant Sciences Research. 2(1): 11-13.

19. Edeoga, H.O., Okwu, D.E. and Mbaebie, B.O. (2005). Phytochemical constituents of some Nigerian medicinal plants. African Journal of Biotechnology. 4(7): 685-688.

20. Rasool, R., Ganai, B. A., Akbar, S., Kamili, A. N. and Masood, A. (2010). Phytochemical screening of Prunella vulgaris L. - an important medicinal plant. Pakistan Journal of Pharmaceutical Sciences. 23 (4): 399-402.

21. Amin Mir, M., Sawhney, S. S. and Jassal, M. M. S. (2013). Qualitative and quantitative analysis of phytochemicals of taraxacum officinale wudpecker. Journal of Pharmacy and Pharmocology. 2(1): $001-$ 005 .

22. Zhou, P. and Regenstein, J.M. (2006). Determination of total protein content in gelatin solutions with the Lowry or biuret assay. Journal of Food Science. 71(8): 474-479. 
23. Sudam, N., Manish, B., Ritesh, M., Sachin, P., Ratnaparkhi, M. P. and Shilpa, C. (2012). Evaluation of various natural suspending agents for its suspending behaviour using paracetamol as model drug for suspension. Asian Journal of Pharmaceutical and Clinical Research. 0974-2441.

24. Mehta, K., Patel., H. H., Patel, N. D., Vora, C. N. and Patel, N. (2010). Comparative evaluation of natural and synthetic superdisintergrant for promoting nimesulide dissolution for fast dissolving technology. International Journal of Pharmacy and Pharmaceutical Sciences. 2(3): 102-103

25. Nair, B. R. and Fahsa K. S. (2013). Isolation and characterization of mucilage from some selected species of Abelmoschus medik (Malvaceae) and their application in pharmaceutical suspension preparation. International Journal of Pharmacy and Pharmaceutical Sciences. 5(1): 398.

26.Singh, A. K., Selvam, R. P. and Sivakumar, T. (2010). Isolation, characterization and formulation properties of a new plant gum obtained from Mangifera indica. International Journal of Research in Pharmaceutical and Biomedical Sciences. 1(2): 35-41.

$$
\begin{aligned}
& \text { 27. عزيز، سلوى ليلو وسلمان، ضحى داود. (2011). استخلاص ودر اسـة بعض الصفات الفيزوكيميائية لصمغ بذور الحلبة. مجلة العلوم }
\end{aligned}
$$

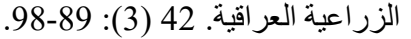

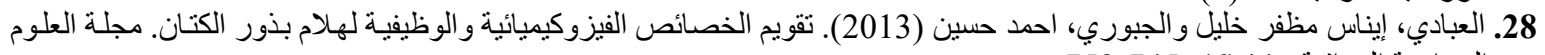

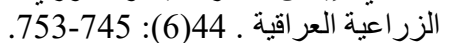

29. Hebert, V. \& Sales, E. (2011). Bulk Density of Powder Made Easy. Brookfild Engineering Labortories, Inc. http:// www.brookfieldengineering.com

30. Emeje, M., Nwabunike, P., Isimi, C., Fortunak, J., Mitchell, J. W., Byrn, S., Kunle, O. and Ofoefule, S. (2009). Isolation, characterization properties of a new plant gum obtained from Cissus refescence. International Journal of Green Pharmacy. 2:16-23.

31. Oyi, A., Shittu, A. and Mahmud, H. (2010). Physicochemical characterization of Acacia sieberiania gum. Indian Journal of Novel Drug Delivery. 2(3): 99-102.

32. Adebowale, K. O., Afolabi, T. A. and Olu-Owolabi, B. I. (2006). Functional physicochemical and retrogradation properties of sword bean (Canavalia gladiata) acetylated and oxidized starch. Carbohydrate Polymers. 65: 93- 102.

33. Zaku, S. G., Aguzue, O. C., Thomas, S. A. and Barminas, J. T. (2009). Studies on the functional properties and the nutritive values of amura plant starch (Tacca involucrata) a wild tropical plant. African Journal of Food Science. 3 (10): 320-322.

34. Valaei, S. R., Hassan-Beygi, M. H., Kianmehr, J. (2012). Mass investigation of avalanche time and carr's index of poultry litter powder as flowability. Cercetari Agronomice in Moldova. 4(152).

35. Mohrig, J. R., Hammond, C. N. and Schatz, P. F. (2010). Techniques in organic chemistry. W. H. Freeman and Company 41 Madison Avenue, New York, Ny 10010 Houndmills, Basingstoke, Rg21 6xs, England.

36. Patel, N. C., Shah, V. N., Mahajan, A. N. and Shah, D. A. (2011). Isolation of mucilage from Cydonia vulgaris Pers. seeds and its evaluation as super disintegrant. Journal of Applied Pharmaceutical Science. 01(04): 110-114.

37. Srichuwong, S., Sunarti, T. C., Mishima, T., Isono, N. and Hisamatsu, M. (2005). Starch from different botanical source. ii: contribution of starch structure to swelling and pasting properties. Carbohydrate Polymers. 62(1): 25-34. 